\title{
Acute myocardial infarction and subclavian artery occlusion in a 41-year-old woman with Behçet's disease: coronary and large vessel arteritis
}

Ho $\underline{\text { So }}^{1}$, MBBS, FHKCP, Man Lung $\underline{Y i p}^{1}$, MBChB, FHKCP

\begin{abstract}
We report the case of a 41-year-old Chinese woman with Behçet's disease (BD) complicated by acute myocardial infarction, requiring inotropic and ventilatory support. Angiography showed critical left anterior descending coronary artery stenosis, a blocked left subclavian artery and left carotid artery stenosis. The patient was successfully treated with a high dose of immunosuppressants, standard anti-ischaemic therapy and percutaneous coronary intervention. Although life-threatening, coronary arteritis is a treatable manifestation of BD. We suggest that the diagnosis of coronary arteritis be considered in patients with BD who present with chest pain. Involvement of other arteries should also be looked out for in these patients.
\end{abstract}

Keywords: acute myocardial infarction, Behçet's disease, coronary arteritis, large vessel arteritis, left subclavian artery occlusion

\section{INTRODUCTION}

Behçet's disease (BD) is a systemic inflammatory disorder of unknown aetiology that is characterised by recurrent oral and genital ulceration, and ocular lesions. It may involve the joints, skin, central nervous system, gastrointestinal tract and cardiovascular system. The prevalence and clinical expression of BD vary geographically - the disease affects people in the Middle and Far East more often than others. ${ }^{(1)}$ Cardiovascular involvement is infrequent and coronary arteritis is very rare. ${ }^{(2)}$ The coexistence of coronary arteritis and large vessel arteritis is even rarer. ${ }^{(3)}$ There are only a few reported cases of coronary arteritis presenting with acute myocardial infarction (AMI) in the literature. ${ }^{(3-8)}$ Herein, we report the case of a middle-aged Asian woman with BD complicated by coronary arteritis and left subclavian artery occlusion who presented with AMI.

\section{CASE REPORT}

A 34-year-old Chinese woman first presented to us in 2001 with recurrent oral and genital ulceration. She also had on-and-off acnelike skin lesions on her body. The patient had a negative pathergy test and no eye symptoms. However, inflammatory markers, such as erythrocyte sedimentation rate (ESR) and C-reactive protein (CRP) level, were found to be elevated. The patient was subsequently diagnosed with $\mathrm{BD}$ and given low-dose colchicine, which helped to improve her symptoms and inflammatory markers. Two years later, in 2003, the patient presented with erythema nodosum (EN) in both shins, together with elevation of inflammatory markers. Treatment with nonsteroidal antiinflammatory drugs was unsuccessful. Azathioprine (AZA) was thus added, with a good treatment outcome seen. In 2006, AZA was stopped as the patient remained asymptomatic, with normal ESR and CRP level. However, the disease flared in 2007 with recurrent $\mathrm{EN}$, oligoarthritis and elevation of inflammatory markers. AZA $75 \mathrm{mg}$ daily was resumed and the disease control improved.
In 2008, the patient collapsed along a street. Upon arrival at the accident and emergency department, she was found to be semiconscious and hypotensive. Electrocardiography (ECG) demonstrated ST segment depression and T-wave inversion in leads V2-4. The patient was intubated and sent to the coronary care unit. Echocardiography revealed hypokinetic anterior and lateral walls with impaired left ventricular systolic function. Creatine kinase and troponin I levels were elevated.

A diagnosis of anterior wall AMI was established. The patient required transient inotropic support. Aspirin, $\beta$-blocker, angiotensin-converting-enzyme inhibitor and low-molecularweight heparin $(\mathrm{LMWH})$ were also administered. The patient's condition improved and she was extubated three days after admission. She reported on-and-off exertional chest pain for two weeks prior to admission. She had no history of smoking or a family history of early cardiovascular diseases. She denied any illicit drug use and her toxicology screening was negative. Her fasting blood glucose and lipid levels were within the respective normal ranges, but ESR and CRP level were found to be elevated. Although the patient's thrombophilia screening was negative and her lower limb pulses were normal, pulses of her left upper limb were found to be weaker than that of her right side, with significant blood pressure difference of $\sim 30 \mathrm{mmHg}$. Bruits were heard over the left carotid and left subclavian regions. Contrast-enhanced computed tomography (CT) of the thorax showed stenosis over the left common carotid and left subclavian arteries, with no sign of aortic dissection. Coronary angiography revealed a critical ostial left anterior descending (LAD) artery stenosis, while other parts of the coronary arteries appeared normal. Aortography performed at the same session revealed a blocked left subclavian artery from the ostium and a tight ostial left carotid artery stenosis. The findings were suggestive of vasculitis affecting the coronary, left subclavian and left common carotid arteries, complicating

'Department of Medicine and Geriatrics, Kwong Wah Hospital, Hong Kong, China

Correspondence: Dr Ho So, Resident Specialist, Kwong Wah Hospital, 25 Waterloo Road, Yau Ma Tei, Hong Kong, China. h99097668@hotmail.com 
BD. Prednisolone $60 \mathrm{mg}$ daily was started, and colchicine $1 \mathrm{mg}$ daily and AZA $125 \mathrm{mg}$ daily were administered. The patient's inflammatory markers normalised shortly and she was discharged.

Upon discharge, the patient again complained of persistent exertional chest pain. Her exercise tolerance was limited to 5 min of level ground walking. She was admitted one month later for persistent chest pain at rest. ECG revealed dynamic ST segment depression in leads V2-3. There was no enzyme leak. The patient's condition was treated as unstable angina with a course of $\mathrm{LMWH}$. Percutaneous transluminal coronary angioplasty (PTCA) and stenting with drug-eluting stents to the left main and LAD arteries were subsequently done. Clopidogrel was administered to the patient, which relieved her chest pain. Repeat echocardiography showed improved anterior wall contraction and left ventricular systolic function.

However, the patient complained of mild left upper limb numbness with on-and-off lightheadedness. Magnetic resonance (MR) angiography confirmed the subclavian steal phenomenon. Percutaneous endovascular balloon angioplasty was done with successful dilation of the subclavian occlusion, and the patient's symptoms improved. The prednisolone dosage was tapered down and maintained at a low dose. The inflammatory markers remained within normal ranges, and the patient has remained well since.

\section{DISCUSSION}

$\mathrm{BD}$ is often considered a multisystemic vasculitis with a remitting and relapsing course. Clinically evident vascular involvement aries from $7 \%$ to $29 \%{ }^{(2)}$ Venous lesions are more common. Large arterial lesions are infrequent and occur in $1.5 \%-2.2 \%$ of all patients with BD. ${ }^{(9)}$ These lesions are usually aneurysmal. Occlusive large arterial diseases rarely occur. Cardiac involvement in BD, although very uncommon, carries a poor prognosis with mortality as high as $20 \%,{ }^{(10)}$ and includes conduction abnormalities, valvular problems, cardiomyopathy, endomyocardial fibrosis with intracardiac thrombi and coronary artery disease.

AMI most often occurs due to atherosclerotic coronary artery disease. ${ }^{(11)}$ However, in our patient who had no traditional cardiovascular risk factors, coronary arteritis leading to vascular endothelial cellular dysfunction has to be considered. The supporting clinical evidence includes the elevation of inflammatory markers, which usually coincide with her disease flares and positive response to high dose of immunosuppressants. The coexistence of subclavian and carotid stenoses also points to a widespread arteritic process. Unfortunately, histology was not available in this case. The common histological abnormalities of vasculitis in BD include lymphocytic and plasma cell infiltration, disrupted internal elastic lamina, aneurysm and occlusive thrombosis. ${ }^{(3)}$ On the other hand, BD has been said to be associated with coagulation and fibrinolytic system disorders, which may lead to myocardial infarction; however, recent work showed that none of the procoagulant factors are clinically significant in BD. ${ }^{(12)}$

To the best of our knowledge, there is only one other case report in the literature describing the coexistence of coronary and large vessel arteritis in the same patient with $\mathrm{BD} .{ }^{(3)}$ In lyisoy et al's report, a 42-year-old Turkish woman had been diagnosed with $\mathrm{BD}$ and pericardial effusion for 14 years. She presented with acute ST elevation and was diagnosed with AMI, consequently receiving thrombolytic therapy. Coronary angiography in this patient showed total occlusion of the LAD artery. Subsequently, the left internal mammary artery was grafted to the LAD artery. Approximately one year after the bypass surgery, digital subtraction angiography of the left subclavian artery, performed due to pain in the chest and left arm, showed total occlusion of the left subclavian artery and stenosis of the segment of the right internal carotid artery distal to the posterior communicating artery. A saphenous vein graft was placed between the left and right subclavian arteries, and the patient's symptoms improved. lyisoy et al concluded that AMI in the patient was due to arteritis and not atherosclerosis, as there was no evidence of atherosclerosis in the biopsies of the arteries obtained during surgery or the presence of cardiovascular risk factors. There are striking similarities between this patient and ours, with the exception being that the occlusive vascular lesions in the former were treated surgically. The immunosuppressive regime employed in lyisoy et $\mathrm{al}^{\prime} \mathrm{s}^{(3)}$ case was, however, not mentioned.

According to the European League Against Rheumatism (EULAR) recommendations for the management of $\mathrm{BD}$, there is no good quality evidence to support the use of anticoagulants, antiplatelets and antifibrinolytics, or guide the choices of immunosuppressants in the management of major vessel disease. ${ }^{(13)}$ Several different therapeutic approaches to manage AMI in patients with BD have been reported in the literature. For instance, Hattori and Kawana described a patient with BD who was successfully treated for myocardial infarction using methylprednisolone 1,000 mg for three days, followed by prednisolone $60 \mathrm{mg}$ daily. ${ }^{(6)}$ The use of primary PTCA or thrombolytic therapy has also been reported. ${ }^{(7)}$ Considering its high mortality, it might be prudent to first treat AMI according to the standard treatment protocol for the condition.

Regarding definitive treatment for occlusive arterial lesions, the optimal approach and timing is largely unknown. It is generally believed that during the acute phase of vasculitis, the inflamed and fragile tissues are difficult to manipulate, with high frequency of complications encountered. Vascular complications following arterial bypass surgery are common in patients with BD - a study found a $24 \%$ rate of graft occlusion and $13 \%$ rate of anastomotic pseudoaneurysm formation. ${ }^{(14)}$ Meanwhile, the longterm outcome of coronary stent implantation in patients with BD is still unknown, and it is yet unclear whether vasculitis may cause more rapid in-stent restenosis. In view of this, medical treatment may be preferred for BD patients with occlusive vascular lesions. If medical treatment fails, surgery or interventional radiology procedures should be performed when the disease is quiescent.

We strongly believe that the AMI and left subclavian artery occlusion in our patient were due to an arteritic process. We also demonstrated successful treatment of the occlusive lesions with endovascular interventions after the disease activity was controlled by immunosuppressive therapies. As coronary arteritis 
is a rare but life-threatening manifestation of $\mathrm{BD}$, we suggest that coronary artery disease be considered in patients with BD who present with chest pain, even in the absence of conventional risk factors. Other concomitant large vessel involvement should be closely investigated, especially when symptoms develop.

\section{REFERENCES}

1. Idil A, Gürler A, Boyvat A, et al. The prevalnce of Behçet's disease above 10 years. The results of a pilot study conducted at the Park Primary Health Care Center in Ankara, Turkey. Ophthalmic Epidemiol 2002; 9:325-31.

2. Park JH, Han MC, Bettmann MA. Arterial manifestations of Behçet disease. AJR Am J Roentgenol 1984; 143:821-5.

3. Iyisoy A, Kursklioglu H, Kose S, et al. Acute myocardial infarction and left subclavian artery occlusion in Behçet's disease: a case report. Mt Sinai J Med 2004; 71:330-4.

4. Schiff S, Moffatt R, Mandel WJ, Rubin SA. Acute myocardial infarction and recurrent ventricular arrhythmias in Behçet's syndrome. Am Heart J 1982; 103:438-40.

5. Schirmer M, Weidinger F, Sandhofer A, et al. Valvular disease and myocardial infarctions in a patient with Behcet disease. J Clin Rheumatol 2003; 9:316-20.
6. Hattori S, Kawana S. Behçet's syndrome associated with acute myocardial infarction. J Nippon Med Sch 2003; 70:49-52.

7. Kosar F, Sahin I, Gullu H, Cehreli S. Acute myocardial infarction with normal coronary arteries in a young man with Behçet's disease. Int J Cardiol 2005; 99:355-7.

8. Harrison A, Abolhoda A, Ahsan C. Cardiovascular complications in Behçet syndrome: acute myocardial infarction with late stent thrombosis and coronary, ventricular, and femoral pseudoaneurysms. Tex Heart Inst J 2009; 36:498-500.

9. Hamza M. Large artery involvement in Behçet's disease. J Rheumatology 1987 ; 14:554-9.

10. Atzeni F, Sarzi-Puttini P, Doria A, et al. Behçet's disease and cardiovascular involvement. Lupus 2005; 14:723-6.

11. Wang $\mathrm{H}$, Eitzman DT. Acute myocardial infarction leads to acceleration of atherosclerosis. Atherosclerosis 2013; 229:18-22.

12. Yazici H, Fresko I, Stuebiger N. Behcet syndrome, relapsing polychondritis, and eye involvement in rheumatid disease. In: Biglsma J, Burmester G, eds. Eular Compendium on Rheumatic Diseases. London: BMJ Publishing Group and European League Against Rheumatism, 2009:357-374.

13. Hatemi G, Silman A, Bang D, et al. EULAR recommendations for the management of Behcet disease. Ann Rheum Dis 2008; 67:1656-62.

14. Hosaka A, Miyata T, Shigematsu H, et al. Long-term outcome after surgical treatment of arterial lesions in Behcet disease. J Vasc Surg 2005; 42:116-21. 